\title{
Ce que le personnage contemporain dit à la critique, dir. R. AUDET et N. XANTHOS
}

\section{Roberta Sapino}

\section{(2) OpenEdition}

\section{Journals}

\section{Edizione digitale}

URL: https://journals.openedition.org/studifrancesi/32496

DOI: 10.4000/studifrancesi.32496

ISSN: 2421-5856

\section{Editore}

Rosenberg \& Sellier

\section{Edizione cartacea}

Data di pubblicazione: 1 août 2020

Paginazione: $462-463$

ISSN: 0039-2944

\section{Notizia bibliografica digitale}

Roberta Sapino, "Ce que le personnage contemporain dit à la critique, dir. R. AUDET et N. XANTHOs», Studi Francesi [Online], 191 (LXIV | II) | 2020, online dal 01 septembre 2020, consultato il 17 septembre 2021. URL: http://journals.openedition.org/studifrancesi/32496 ; DOI: https://doi.org/10.4000/studifrancesi. 32496

Questo documento è stato generato automaticamente il 17 septembre 2021.

\section{(c) (i) (9)}

Studi Francesi è distribuita con Licenza Creative Commons Attribuzione - Non commerciale - Non opere derivate 4.0 Internazionale. 


\title{
Ce que le personnage contemporain dit à la critique, dir. R. AUDET et $\mathrm{N}$. XANTHOS
}

\author{
Roberta Sapino
}

\section{NOTIZIA}

Ce que le personnage contemporain dit à la critique, dir. R. AUDET et N. XANTHOS, Paris, Presses Sorbonne Nouvelle, 2019, 213 pp.

1 Être de papier, être d'action, être de système: se lo status del personaggio romanzesco rimane oggetto di dibattito (non solo: di un dibattito che si riattualizza incessantemente, e che sempre si arricchisce di elementi nuovi e nuove posture) almeno tanto quanto lo rimane, osservano René AUDET e Nicolas XANTHOS nell'Introduction (pp. 7-13), l'eredità delle teorie strutturaliste, lo scopo di questo ricco volume non è sostenere una di queste tre posizioni, quanto piuttosto «reprendre le dialogue, redire la nécessité théorique, et se remettre à la tâche». Le principali teorie che a oggi strutturano il dibattito sono nate dall'osservazione di un corpus di romanzi del xIx, o al più tardi del primo xx secolo, ma qual è la loro attualità oggi? Come possono aiutarci a comprendere il romanzo contemporaneo? Nel volume, queste domande sono affrontate da tre punti di vista distinti, ma - significativamente - spesso messi in contatto attraverso richiami, citazioni, discorsi sviluppati in maniera trasversale: così, mentre i sottotitoli delle tre sezioni annunciano une perspective poétique, une perspective pragmatique, une perspective culturelle, l'impressione che deriva dalla lettura d'insieme è che il maggior valore del lavoro di Audet e Xanthos risieda nella capacità di mettere proficuamente in dialogo sguardi diversi.

2 La prima sezione, intitolata «De nouvelles configurations pour représenter l'humain», è inaugurata dal contributo di René Audet, il quale osserva come la progressiva «déroute» del personaggio nel romanzo contemporaneo (lo studioso si sofferma su testi 
di Sarraute, Chevillard, Vila-Matas, Bouchard) sia da considerare come il sintomo di un ripensamento globale del genere romanzesco e dei suoi orizzonti di scopo: la performance discorsiva, più che la narrazione, diviene portatrice di senso (Un personnage, s'il le faut. Splendeurs et misères de la pantomime romanesque contemporaine, pp. 17-27). Tara COLLINGTON analizza le dinamiche sociali e dialogiche che intervengono nella costruzione dei personaggi in due romanzi di Hélène Lenoir, Le Répit e La Crue de juillet. Avvalendosi delle teorie di Michail Bachtin e Alan Palmer, la studiosa elabora e mette alla prova una metodologia ibrida in cui l'analisi letteraria si fonde produttivamente con la narratologia cognitiva (De la polyphonie à la narratologie cognitive: la construction du personnage dans l'univers romanesque d'Hélène Lenoir, pp. 29-45).

3 In Entre péremption et préemption: aspects du personnage contemporain, Frank WAGNER smentisce invece le idées reçues che vorrebbero il personaggio morto per sempre sotto i colpi dei nouveaux romanciers per poi dimostrare, attraverso alcuni casi rappresentativi di tendenze contemporanee, che se il personaggio sopravvive è in virtù della sua problematicità e della sua attitudine alla metamorfosi - caratteristiche che lo rendono un valido «révélateur épistémologique» (pp.47-59). Ben cosciente delle accuse di naïveté che potrebbe attirare, nel contributo intitolato $Y$ a de la joie, un suicide et des espions. Psychologie du personnage dans "L'Adieu à Stefan Zweig" de Belinda Cannone, Nicolas Xanthos recupera il concetto di psicologia del personaggio e, sottolineando l'importanza di indagare il rapporto tra personaggio e struttura narrativa, propone un'ipotesi coraggiosa: "la littérature contemporaine comme mode spécifique de rationalité» (pp. 61-71). Conclude la sezione il contributo di Marie-Hélène VOYER: attraverso l'analisi di Deux Bêtes à l'intérieur di Nicolas Bouyssi, la studiosa mette in luce la "poétique de la discrétion» che caratterizza i personaggi e dimostra come la discrezione possa costituire una forma di dissidenza fondata sul rovesciamento dei dispositivi della "società del controllo" («Il faut avoir la politesse d'être heureux». Formes et paradoxes de la discrétion du personnage chez Nicolas Bouyssi, pp. 73-84).

4 La seconda sezione è intitolata «Refigurer l'humain, entre écriture et lecture». In Le 'credit crunch' de la démocratie. Personnage de fiction et idole de papier mâché (dans "Résister ne sert à rien" de Walter Siti), Emmanuel Bouju sviluppa l'idea che il genere del romanzo, luogo dello sdoppiamento finzionale di sé e della sperimentazione di diversi "possibili", sia ad oggi uno degli ultimi baluardi dello scambio democratico (pp. 87-94). Bertrand GERVAIS, nel suo intervento intitolato Chet Baker est une figure. Personnages conceptuels et fiction critique chez Enrique Vila-Matas (pp. 95-106), scompone per meglio comprenderlo l'ingranaggio complesso nel quale si innestano i personaggi - allo stesso tempo letterari e concettuali - di Chet Baker pense à son art: uno spazio nel quale è la letteratura stessa ad essere messa in discussione. Il contributo di Fanny BARNABÉ, Julie DELBOUILLE e BjörnOlav Dozo (Immersion et réflexivité. L'avatar au coeur de la construction de la posture vidéoludique, pp. 107-120) ci conduce nel mondo dei videogiochi. Ribadita l'insufficienza dell'opposizione tra reale e virtuale per comprendere l'esperienza videoludica e messe in prospettiva alcune interpretazioni eccessivamente vaghe o intuitive della nozione di immersione, gli autori osservano come la riflessività non sia da intendere in opposizione all'esperienza immersiva, ma piuttosto come una sua condizione necessaria. Sophie BEAUPARLANT si sofferma poi su un personaggio molto particolare non solo perché recente, ma perché ontologicamente fondato sulla commistione di finzione e realtà: lo youtuber. I canali Solange te parle e Cyprien compongono il corpus di uno studio che si avvale degli strumenti teorici della critica cinematografica, della 
narratologia e della semiotica (Personnages du web: sortir de l'écran pour exister, pp. 121-131).

5 Nella terza sezione, intitolata «Le personnage comme interface des formes de vie», Charline PLUVINET si interroga sui personaggi la cui struttura deriva dalla commistione tra visione antropomorfica e costruzione puramente testuale, soffermandosi in particolare su alcuni romanzi di Roth, Vila-Matas, O'Brien e Delaume in cui l'interrogazione identitaria non si sviluppa solo sul piano tematico, ma contribuisce a ridefinire le modalità narrative (Identité troublée du personnage: permanence et rupture dans les narrations fictionnelles de soi, pp. 135-148). Preoccupazioni analoghe sono sviluppate anche in "Mutatis mutandis". Le personnage littéraire à l'épreuve du vivant (pp. 149-157), dove Bruno BLANCKEMAN osserva alcune costruzioni "paradossali" del personaggio romanzesco del xxI secolo (per esempio in Darrieussecq, Louis, Houellebecq e altri). I motivi della riparazione, della mutazione, della labilità costituiscono, suggerisce l'autore, chiavi interpretative importanti per capire il romanzo contemporaneo senza negare le linee di continuità con le sperimentazioni precedenti. Anne Martine PARENT mette in luce come in Ladivine di Marie NDiaye la porosità tra l'umano e l'animale sia alla base di un discorso antispecista nel quale il cane è elevato a salvatore dell'uomo: solo grazie all'animale l'uomo prende coscienza della propria vulnerabilità e si reinserisce in un sistema di circolazione degli affetti di cui il cane è garante (Dans la peau d'un chien. L'animal au secours de l'humain dans "Ladivine" de Marie NDiaye, pp. 159-172). La commistione tra umano e non-umano, pur in forma molto diversa, è centrale anche nel saggio intitolato Lorsque les Hubots et Ava se rebellent... Le personnage-cyborg féminin du petit et du grand écran (pp. 173-187), nel quale Julie BEAULIEU analizza la serie televisiva Real Humans/100\% Human e il film Ex Machina alla luce di una domanda: «le cyborg féminin est-il un outil aussi subversif que la critique féminine le prétend?».

6 Conclude il volume la ricca Bibliographie, utilmente suddivisa nelle sezioni Corpus, Références critiques, Ludographie e Médiagraphie. 